\section{UK withdrawal from INTEGRAL}

SIR - The United Kingdom's withdrawal from the European Space Agency (ESA)'s INTEGRAL mission (Nature 373,$459 ; 1995$ ) is not just a story of the offended interests of some British scientists: it is a tale of Britain turning its back on a European agreement, leaving fellow ESA member states to pick up the pieces. Although playing the role of prima inter pares, Britain did not "initially propose" INTEGRAL, as stated in the article. Rather, it was a European, and indeed worldwide, collaboration, to propose the INTErnational Gamma Ray Astrophysical Laboratory.

Indeed, to describe the instrument, proposed by A. J. Dean of the University of Southampton as principal investigator, as a "UK/Italian project" neglects the important French contribution, as well as the Spanish and Finnish/Norwegian/ Swedish participation in that proposal.

As to the background, INTEGRAL was selected by ESA's science programme committee in June 1993 as its next ' $M$ ' (medium class) mission. Britain voted in favour; to quote from the minutes: "The UK delegation thoroughly approves the recommendation of INTEGRAL, which had come at a particularly good time". A few weeks earlier, there had been a public debate at the Royal Institution in London on UK involvement in the next ESA science mission. INTEGRAL was selected from a number of candidates, partly on the strength of the proposed contribution to the payload by British groups. Costs were of course discussed. Indeed, aware of the potential problems, Roger Bonnet, director of ESA's space science programmes, had asked for particular accuracy and transparency in this exercise. The UK national delegates were therefore fully aware of the cost implications of the payload when voting so enthusiastically in favour of INTEGRAL.

Britain's recent decision to withdraw therefore appears to be the result of a misunderstanding (or management blunder) between scientists who put forward a mission in good faith and a financing body that is now reneging on an agreement with the rest of Europe. Perhaps the really interesting story is the one behind this spectacular change in the UK position.

There is an interesting precedent. About 25 years ago, Britain withdrew from an earlier European science mission, again a gamma-ray mission (COS-B) in which the University of Southampton was to have played a key role. The result was a damaging blow for many years to gammaray astronomy in Britain and a very successful mission for the rest of Europe. Historia magistra vitae?

As to picking up the pieces, it is now obvious that the other ESA nations will have to do their part. Italy is cautiously trying to do so, essentially because INTEGRAL exists, not because of "economic logic". Italy and others are trying to save a mission crucial to ESA's science programme that has been put in grave danger by Britain's withdrawal.

GlovannI F. BIgnaml

8 viale Lombardia,

20131 Milano, Italy

SIR - Your article on the ESA science programme and the United Kingdom's difficulties in participating in INTEGRAL, the medium-class mission approved last year, raises some important issues for the future of space science in the United Kingdom. This mission was originally proposed from the United Kingdom and the UK team played a leading role in the studies that led to its selection against severe competition. Technology development has taken place in the United Kingdom over a 10-year period in preparation for this mission and it is not surprising in consequence that it was rated top priority here for the mission selection and that the UK delegation to the ESA science programme committee then voted accordingly last year. The likely costs of UK participation were well known and have not changed substantially.

If UK scientists cannot, with this degree of careful preparation, participate in the ESA programme, one wonders in what circumstances they will be able to do so. Ken Pounds, the chief executive of the Particle Physics and Astronomy Research Council (PPARC), has offered the explanation that his new research council inherited a good programme but an inadequate budget from its predecessor, the Science and Engineering Research Council. From his point of view that is no doubt all there is to say, but for those of us who expected to help build and launch the INTEGRAL instruments it is not convincing, nor should it be for those who hope to play similar roles in the future missions in ESA's programme. The UK companies that should have had a major part in the design and construction of advanced gamma-ray and optical instrumentation have reason to feel particularly dismayed. The funding of the domestic space programme has for a number of years been subject to recurrent crises resulting from a combination of the long-term nature of the programme and the failure of the research councils to provide secure planning for funding on the necessary timescale. It is essential that PPARC, in conjunction with the British National Space Centre, which has the responsibility for national planning in space matters, should find a solution to this problem.

The United Kingdom will, in any case, contribute at significant cost to the INTEGRAL mission through its subscription to the ESA science programme. At issue here is the return to UK astronomy through access to the observations INTEGRAL will make and through the stimulation of a healthy UK gamma-ray community.

J. L. Culhane

Mullard Space Science Laboratory,

Holmbury St Mary,

Dorking, Surrey RH5 6NT, UK

A. J. Dean

University of Southampton,

Southampton, UK

\section{A. P. Willmore}

Space Research Group,

University of Birmingham,

Edgbaston,

Birmingham B152TT, UK

\section{Common noun}

SIR - Hi-jacking names may be more common and more aggressive today than most of us think (Nature 373, 370; 1995). In Germany we have another shining example. The pet food company Effem $\mathrm{GmbH}$, Verden/Aller has registered the word 'Pedigree' as a trademark. It is used to market food for dogs. The word 'pedigree' meaning the ancestry of an individual is widely used among geneticists, and thousands of articles have been published using this word. A nice feature of the noun is that it is identical in several languages, for example English, Spanish, French, and German. Furthermore, the word is used not only by the scientific community but also by practical breeders of any kind. More stringent rules should apply when trademarks are registered.

\section{Hermann Swalve}

Institut für Tierzucht

und Haustiergenetik,

Georg-August-Universität,

Albrecht-Thaer-Weg 3,

D-37075 Göttingen, Germany

\section{Keep off politics}

SIR - The Smithsonian wouldn't have got itself into this mess if it had stuck to its last (Nature 373, 371; 1995).

By all means debate the Enola Gay and the dropping of the A bomb, but not at the Smithsonian's Air and Space Museum: it is an entirely inappropriate place to do so. This Mecca to aviation is the world's supreme technological showplace and the crown jewel of the Washington tourist scene; it has never been previously tarnished with contentious political messages attached to an exhibition and nor should it be now.

\section{George Fulford}

218 Reed Circle,

Mill Valley, California 94941, USA 